\title{
NEW LIPID EMULSIONS FOR THE PN OF PRETERM INFANT: IMPACT ON ARA, EPA AND DHA STATUS
}

\author{
R. D'Ascenzo ${ }^{1}$, S. Savini ${ }^{1}$, G. Serpentini ${ }^{1}$, I. Burattini ${ }^{1}$, A. Bartoli ${ }^{1}$, C. Biagetti ${ }^{1}$, M.P. Bellagamba ${ }^{1}$, F. \\ Staffolani ${ }^{1}$, P.E. $\operatorname{Cogog}^{2}$, V.P. Carnielli ${ }^{1}$ \\ ${ }^{I}$ Neonatology, Polytechnical University of Marche, Ancona, ${ }^{2}$ DMCCP, Pediatric Hospital "Bambino Gesù", \\ Rome, Italy
}

Background and aims: New lipid emulsions containing fish oil (FO) have recently became available and they could be proven to be a good source of Arachidonic Acid (ARA) and Docosahexaenoic Acid (DHA).We compared in preterms the impact of 2 FO containing lipid emulsions and 3 conventional preparations on plasma phospholipid fatty acids (PLFA) status.

Methods: Seventyfive preterms $<1250 \mathrm{~g}$ were randomized (15 in each groups) to receive PN with MS (50\%MCT,50\%soy),OS (80\%olive, $20 \%$ soy), SO (100\%soy),FMS (10\%FO,50\%MCT,40\%soy) and FOMS $\left(15 \% \mathrm{FO}, 25 \%\right.$ olive, $30 \% \mathrm{MCT}, 30 \%$ soy). The IV lipid intake was increased in a stepwise fashion to $2,5 \mathrm{~g} \cdot \mathrm{kg}^{-}$ ${ }^{1} \cdot \mathrm{d}^{-1}$ by PN d7.All infants were on minimal enteral feeding.Plasma PLFA were determined at $\mathrm{d} 7$.

Results: ARA and DHA intakes $\left(\mathrm{mg} \cdot \mathrm{kg}^{-1} \cdot \mathrm{d}^{-1}\right)$ on PN $\mathrm{d} 7$ are in table2. ARA, EPA and DHA of plasma PL $(\mathrm{Mol} \%)$ on PN d7 are in table2.

\begin{tabular}{|l|c|c|c|c|c|c|}
\hline & MS & OS & SO & FMS & FOMS & p \\
\hline ARA & $5.7 \pm 1.3^{\alpha}$ & $6.8 \pm 1.3^{\alpha}$ & $6.0 \pm 1.1^{\alpha}$ & $9.8 \pm 1.2^{\beta}$ & $9.1 \pm 1.7^{\beta}$ & 0.000 \\
\hline DHA & $4.7 \pm 1.1^{\alpha}$ & $3.6 \pm 0.6^{\alpha}$ & $1.4 \pm 0.3^{\alpha}$ & $56.5 \pm 4.1^{\beta}$ & $47.8 \pm 5.6^{\beta}$ & 0.000 \\
\hline
\end{tabular}

[Table $*$ ]

\begin{tabular}{|l|c|c|c|c|c|c|}
\hline \multicolumn{1}{|c|}{} & MS & OS & SO & FMS & FOMS (n=15) & p \\
\hline ARA & $11.8 \pm 1.3^{\alpha}$ & $10.9 \pm 1.2^{\alpha}$ & $11.9 \pm 1.7^{\alpha}$ & $10.5 \pm 1.3^{\alpha}$ & $9.7 \pm 1.1^{\beta}$ & 0.000 \\
\hline EPA & $0.3 \pm 0.1^{\alpha}$ & $0.2 \pm 0.3^{\alpha}$ & $0.3 \pm 0.1^{\alpha}$ & $1.6 \pm 0.4^{\beta}$ & $1.4 \pm 0.3^{\beta}$ & 0.000 \\
\hline DHA & $2.4 \pm 0.3^{\alpha}$ & $2.0 \pm 0.4^{\alpha}$ & $2.1 \pm 0.3^{\alpha}$ & $2.8 \pm 0.3^{\beta}$ & $3.0 \pm 0.4^{\beta}$ & 0.000 \\
\hline
\end{tabular}

[Table $2 *]$

[*Mean \pm SD,ANOVA,different superscripts indicates $\mathrm{p}<0.01$ by Bonferroni]

Conclusion: Both the $10 \%$ and $15 \%$ FO containing emulsions ensured intrauterine DHA intakes. Plasma ARA PL was lower,Eicosapentaenoic Acid (EPA) and DHA higher in FO groups compared with the standards.The potential benefits on neurodevelopment and on inflammation need large randomized clinical trials. 